\title{
The steering gear control system based on Arduino
}

\author{
Huaimeng Gui \\ Information Engineering Department, Shaanxi Polytechnic Institute, Shaanxi, Xianyang, China \\ Corresponding author:304913013@qq.com
}

Keywords: Arduino, control system, steering gear

\begin{abstract}
The steering gear is a key component to control the robot localization and motion. In this paper, the working principle of the steering gear was introduced. In experiment, two methods of using Arduino controller realized a single steering gear working, and made a comparative analysis of these methods. This conclusion provides a theoretical basis for using the Arduino to control multiple steering gears.
\end{abstract}

\section{Introduction}

Robots are the machine devices that perform work automatically. They can not only accept human commands, but also run pre-programmed programs. And they can also act according to the principle program formulated by artificial intelligence technology. Robots are the product of integration of cybernetics, mechanical electronics, computers, materials and bionics. At present, robots have important applications in industrial, medical, agricultural and even military fields. Robots have many articulated machines, each of which called a degree of freedom. The general body has more than ten degrees of freedom so as to ensure the flexibility of movement. In the robot body, we usually use steering gear as the connecting part of each joint, which can complete the positioning and movement of each joint ${ }^{[1-2]}$.

Arduino is an open source electronic prototype platform that is convenient, flexible and easy to use, including hardware (various types of Arduino boards) and software (Arduino IDE) ${ }^{[3]}$. It is built on the open source simple I / O interface version and has a processing / wiring development environment using Java and C languages. It mainly includes two main parts: the hardware part is an Arduino circuit board that can be used for circuit connection. The other is Arduino ide, the program development environment in the computer. The advantages of using Arduino to create or develop products are obvious, such as cross-platform, simple and clear development, openness, and community and third party support. Therefore, more and more professional hardware developers have used Arduino to develop projects and products ${ }^{[4]}$.

The traditional steering gear is mainly driven by a microcontroller system. A robot needs multiple steering gears to work at the same time, but the microcontroller system cannot drive multiple steering gears to work well. Arduino provides a <servo.h> library, which makes the control of steering gear more convenient. This paper gives two methods to control the rotation of a steering engine by using Arduino circuit board, which provides a theoretical basis for controlling multiple steering engines to work at the same time by using Arduino circuit board. 


\section{Working principle}

Steering gear is a kind of position servo driver, which is mainly composed of casing, circuit board, coreless motor, gear and position detector, as shown in figure 1. Its working principle is that a signal is sent to the steering gear by a receiver or a single chip microcomputer. A reference circuit is arranged inside the receiver and generates a reference signal with a period of $20 \mathrm{~ms}$ and a width of $1.5 \mathrm{~ms}$. The obtained DC bias voltage is compared with the voltage of the potentiometer to obtain a voltage difference output. The IC on the circuit board determines the direction of rotation, then drives the coreless motor to start rotation, transmits power to the swing arm through the reduction gear, and sends back signals from the position detector to determine whether the positioning has been reached. It is suitable for those control systems that require constant change in angle and can be maintained. When the rotation speed of the motor is constant, the potentiometer is driven to rotate through the cascade reduction gear, so that the voltage difference is zero, and the motor stops rotating. Generally, the rotation angle of steering gear ranges from 0 degrees to 180 degrees.

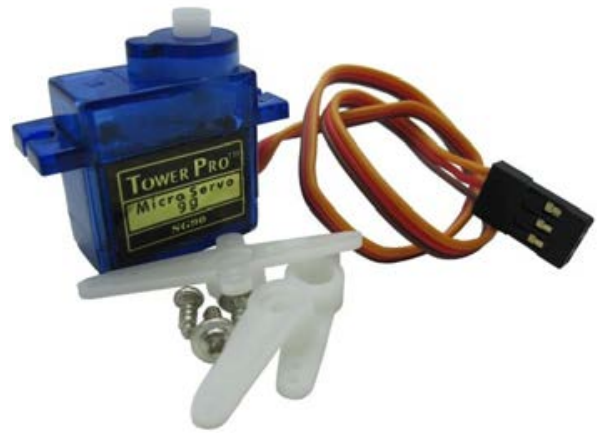

Figure 1. Steering gear physical device

There are many specifications for steering gear, but all steering gears have three external wires, which are respectively distinguished by brown, red and orange colors. Due to the different brands of steering gear, the colors will also be different. Brown is the ground wire, red is the positive wire of the power supply, and orange is the signal wire.

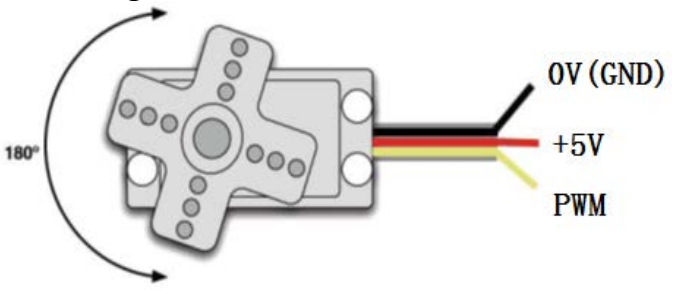

Figure 2. Steering gear three-wire interface

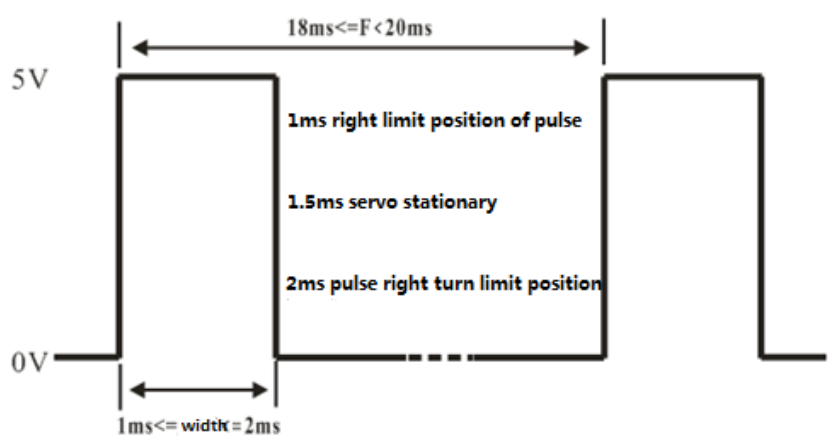

Figure 3. Schematic diagram of steering gear angular rotation principle 
The rotation angle of the steering gear is realized by adjusting the duty ratio of the PWM (pulse width modulation) signal. The period of the standard PWM (pulse width modulation) signal is fixed at $20 \mathrm{~ms}(50 \mathrm{~Hz})$. Theoretically, the pulse width distribution should be between $1 \mathrm{~ms}$ and $2 \mathrm{~ms}$, as shown in Fig. 3. However, in fact, the pulse width can range from $0.5 \mathrm{~ms}$ to $2.5 \mathrm{~ms}$, and the pulse width and the servo angle can range from 0 to 180 degrees.

\section{Experiment}

There are two ways to control the steering gear with Arduino. One is to generate square waves with different duty ratios through the common digital sensor interface of Arduino, and simulate to generate PWM signals to position the steering gear. The other is to control the steering gear directly by using the servo function brought by Arduino. The advantage of this control method is that the program is written, but the disadvantage is that only two steering gears can be controlled. The function brought by Arduino can only use the digital 9 and 10 interfaces, and its connecting circuit is shown in figure 4. Because of limited driving capacity of Arduino, it needs external power supply when more than one steering gear needs to be controlled.

Method 1: The servo is rotated to the angle number position corresponding to the number by the user, and angle printing is displayed on the screen.

int servopin=9 ; // Define the digital interface 9 is connected with the signal line of the servo steering engine

int myangle ; // Define angle variables

int pulsewidth ; // Define pulse width variables

int val ;

void servopulse(int servopin, int myangle) // Define a pulse function

pulsewidth $=($ myangle*11) +500 ; // Converts the angle to a pulse width value

digitalWrite (servopin, HIGH) ; // Defines the steering gear interface as high level

delayMicroseconds (pulsewidth) ; // Number of microseconds for delayed pulse width value

digitalWrite (servopin, LOW) ; // Defines the steering gear interface as low level

delay (20-pulsewidth / 1000) ;

\}

void setup()

\{

pinMode(servopin, OUTPUT) ; // Setting the steering gear interface as the output interface

Serial.begin(9600); // Connect to serial port with baud rate of 9600

Serial.println ("servo=0_seral_simple ready" ) ;

\}

void loop()

\{

val=Serial.read() ; // Read the value of the serial port

if(val $>$ '0'\&\&val<='9')

\{

val=val-'0';

val=val*(180/9) ;

Serial.print("moving servo to ") ;

Serial.print(val,DEC) ;

Serial.println();

for(int $\mathrm{i}=0 ; \mathrm{i}<=50 ; \mathrm{i}++) / /$ Give the steering gear enough time to turn to the specified angle.

\{ 
servopulse(servopin,val) ; // Refer impulse function

\}

\}

\}

Method 2:Use Arduino's own servo function and its statement. Connect the steering gear to the digital 9 interface.

\#include $<$ Servo.h $>$

Servo myservo ; // Define the steering gear variable name

void $\operatorname{setup}()$

\{

myservo.attach(9) ; // Define steering gear interface

\}

void $\operatorname{loop}()$

\{

myservo.write(90); // Set the steering gear rotation angle \}

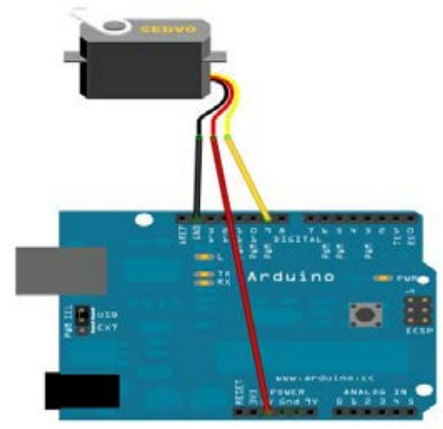

Figure 4. Schematic diagram of arduino control steering gear connection

\section{Conclusion}

Firstly, this paper introduces the working principle of steering gear in detail. In the experiment, the arduino control board is used to control several single steering engines. At the same time, two methods for controlling steering gear are introduced. One is to generate square waves with different duty ratios through arduino's common digital sensor interface, and simulate to generate PWM signals for steering gear positioning; The other is to control the steering gear directly by using the servo function brought by arduino. the two methods are compared and analyzed. This provides a theoretical basis for arduino to control multiple steering engines.

\section{References}

[1] P. L. Peng, Y. J. Feng, Z. Y. Liu, Automation and Instrumentation, 31, 1(2016)

[2] B. H. Yuan, Z. Dong, Journal of Anhui Agri., 40, 5049(2012).

[3] Buechley, L. Eisenberg, Michael. IEEE pervas. comput. , 7, 12(2008).

[4] W. G. Liu, H. B. Wang, Electronic Design Engineering, 22, 150 (2015) 